\title{
Aplicación de RPC-PLFR en el diagnóstico de micobacterias no tuberculosas
}

\author{
Sergio L. Yzquierdo S., Liliam Mederos C., Alexis Díaz G., Miguel Echemendia F. y Ernesto Montoro C.
}

\section{Application of PCR-RFLP in the diagnosis of non-tuberculous mycobacteria}

The amplification of a fragment from hsp65 gene by polymerase chain reaction (PCR) followed by restriction fragment length polymorphism (RFLP) analysis with BstEII and HaeIII restriction enzymes has demonstrated to be very useful for identification of Non-Tuberculous Mycobacteria (NTM). The biochemical tests as well as the PCR-RFLP were carried out in 13 reference strains and 46 strains received in the laboratory. The results by biochemical tests were available in 4-6 weeks whereas the PCR-RFLP only required 48 hours. In both methods, Mycobacterium intracellulare, M. kansasii and M. fortuitum were the most frequently detected species. The PCR-RFLP method is fast, cheap and simple. Its application in Reference Laboratories could be very useful for diagnosis of NTM.

Key words: Non-tuberculous mycobacteria, polymerase chain reaction, restriction fragments length polymorphism, biochemical test.

Palabras claves: Micobacterias no tuberculosas, reacción de polimersa en cadena, polimorfismo de la longitud de los fragmentos de restricción, pruebas bioquímicas.

\section{Introducción}

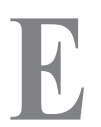

n 1882, Robert Koch dio a conocer al mundo al bacilo Mycobacterium tuberculosis como el agente causal de la tuberculosis (TBC). Sin embargo, se sabe actualmente que existen más de 100 especies dentro del género Mycobacterium con características fenotípicas y bioquímicas bien disímiles a las especies pertenecientes al complejo M. tuberculosis ${ }^{1}$. Estos bacilos "atípicos", conocidos como micobacterias no tuberculosas (MNT), son de gran importancia clínica en pacientes inmunocomprometidos. La detección rápida y eficaz de estas micobacterias resulta crucial para la adopción de una correcta terapia específica en los pacientes, permitiendo su pronta recuperación y evitando una posible diseminación de la enfermedad ${ }^{2,3}$.

Por tal motivo, numerosos métodos han sido diseñados para el diagnóstico de las MNT. En ellos se trata de lograr una identificación precisa de las mismas, para poder aplicar un tratamiento eficaz en los pacientes, ya que estas especies poseen diferentes patrones de susceptibilidad frente a los fármacos antituberculosos ${ }^{2,3}$.

Muchos laboratorios de referencia realizan la identificación de especies micobacterianas por análisis de las características fenotípicas y bioquímicas, las cuales resultan en ocasiones laboriosas y demoran de 3 a 6 semanas en obtener los resultados ${ }^{4}$.
Los métodos basados en el análisis lipídico, tales como la cromatografía líquida de alta resolución, la cromatografía de capa delgada y la cromatografía de gas líquido, han sido aplicados en la identificación de especies micobacterianas. Estos procedimientos suelen ser engorrosos, no son lo suficientemente discriminativos y caros, sólo accesibles para algunos laboratorios clínicos $^{5-8}$. Las técnicas serológicas pueden ser de gran utilidad en determinadas ocasiones pero, tanto su especificidad como sensibilidad son insatisfactorias ${ }^{9}$.

Las pruebas comerciales Accuprobe ${ }^{\circledR}$ (Gen-Probe, Inc., San Diego, California, EUA) e INNO-LiPA (Mycobacteria, Inmunogenetics, Bélgica) son muy confiables pero extremadamente costosas para países de bajos recursos y no aplicables para toda la gama de especies micobacterianas $^{6}$.

Hance y cols, reportaron en 1989 la amplificación de un fragmento del gen de la proteína de estrés térmico de peso molecular $65 \mathrm{kDa}$ ( $h s p 65)$, el cual acoplado con pruebas específicas para especies permitía la identificación de micobacterias provenientes de muestras clínicas. Posteriormente, Plikaytis y cols en 1992 y Telenti y cols en 1993, perfeccionaron el método mediante la amplificación de dicho fragmento por RPC seguido del análisis del polimorfismo de la longitud de los fragmentos de restricción (PLFR) originados por la acción de las enzimas BstEII y HaeIII ${ }^{10-12}$.

\author{
Instituto de Medicina Tropical \\ "Pedro Kourí" (IPK). La Habana, \\ Cuba \\ Laboratorio Nacional de Referencia \\ e Investigación de Micobacterias y \\ Tuberculosis \\ Este trabajo fue financiado por el \\ proyecto INCO-Acción Concertada \\ Improve Diagnosis and Drug \\ Resistance Detection in Latin \\ America, ICA4-CT-2001-10087. \\ Recibido: 25 abril 2007 \\ Aceptado: 13 agosto 2007
}

Correspondencia a: Ernesto Montoro Cardoso emontoro@ipk.sld.cu 
En el presente trabajo se aplicó la RPC-PLFR como una herramienta rápida en el diagnóstico rápido de MNT en el Laboratorio Nacional de Referencia e Investigación de Micobacterias y Tuberculosis del Instituto de Medicina Tropical "Pedro Kourí" (LNRTBIPK).

\section{Material y Método}

Cepas. Se estudiaron trece cepas de referencias correspondientes a 12 especies de micobacterias y pertenecientes a la colección del LNRTB-IPK: $\mathrm{Myco}$ bacterium smegmatis (ATCC 609), Mycobacterium fortuitum (Denver Smith 6196), Mycobacterium avium (ATCC 25291), Mycobacterium peregrinum (TMC 1547, Turtle), Mycobacterium gastri (TMC 1456), Mycobacterium nonchromogenicum (ATCC 19350), Mycobacterium scrofulaceum (ATCC 19981) Mycobacterium intracellulare (serotipo 18, Denver 6450-204), Mycobacterium flavescens (Denver 2611), Mycobacterium kansasii (Denver-Cape 17), Mycobacterium simiae I (Denver 1595), Mycobacterium simiae II (Denver w-55) y Mycobacterium tuberculosis $\mathrm{H}_{37} \mathrm{R}_{\mathrm{V}}$ (ATCC 35838).

Así mismo, se estudiaron 46 MNT aislados en pacientes inmunocomprometidos (8) y portadores del virus de inmunodeficiencia humana (30). De los 38 pacientes, 26 manifestaban síntomas de una enfermedad pulmonar, en su mayoría tos crónica, acompañada en ocasiones de pérdida de peso, fatiga, debilidad y fiebre. En los restantes 12 pacientes, las muestras fueron obtenidas por exudados o biopsias. Todas las muestras fueron colectadas por el Centro Provincial de Higiene y Epidemiología de Ciudad de La Habana, en el período comprendido entre enero de 2005 y abril de 2006 e identificadas por el LNRTB - IPK en septiembre del propio año.

Pruebas bioquímicas. A las 46 cepas recibidas en el laboratorio se les realizó una batería de pruebas fenotípicas y bioquímicas según la metodología descrita por Vestal ${ }^{13}$ así como Kent y cols ${ }^{14}$, incluyendo: crecimiento a 25,37 y $45^{\circ} \mathrm{C}$; evaluación de producción de pigmentos; crecimiento en presencia de isoniacida; tolerancia al $\mathrm{NaCl} 5 \%$; crecimiento en hidroxilamina; catalasa semicuantitativa; reducción de nitratos a nitritos; ureasa; pirazinamidasa; fosfatasa ácida; arilsulfatasa; $\beta$-galactosidasa, hidrólisis del Tween 80 y fuente de carbono (este último para micobaterias de crecimiento rápido).

Preparación de la muestra para la RPC-PLFR. La preparación de las muestras se realizó según el trabajo descrito por Telenti y cols ${ }^{12}$. Se tomó una asada de colonia micobacteriana y se resuspendió en $400 \mu \mathrm{L}$ de TE (Tris-HCl $10 \mathrm{mM}$, EDTA $1 \mathrm{mM}, \mathrm{pH} 7,4$ ) en viales de $1,5 \mathrm{~mL}$ y luego fueron sometidos a $80{ }^{\circ} \mathrm{C}$ por $30 \mathrm{minu}-$ tos. Las muestras fueron centrifugadas a $13.000 \mathrm{rpm}$ durante 10 minutos y el sobrenadante se conservó a $-20{ }^{\circ} \mathrm{C}$, siendo suficientes $5 \mu \mathrm{L}$ del mismo para la RPC. Como control negativo del ensayo se empleó $5 \mu \mathrm{L}$ de agua destilada estéril.

$R P C-P L F R$. Fue aplicada tanto en las 13 cepas de referencia como a las 46 cepas recibidas en el laboratorio. Se utilizaron los cebadores Tb11 (5'-ACCAA CGATGGTGTGTCCAT-3')y Tb12 (5'-CTTGTCGAAC CGCATACCCT-3'), descritos por Telenti y cols ${ }^{12}$ y Devallois y cols ${ }^{15}$, para amplificar un fragmento de aproximadamente 439 pares de bases (pb) del gen hsp65 en 13 cepas de referencia. La mezcla de reacción contenía a ambos cebadores en una cantidad de 25 pmoles, $5 \mu \mathrm{L}$ de tampón de reacción (10 mM Tris- $\mathrm{HCl}, 50 \mathrm{mM}$ $\mathrm{KCl}, 0,1 \%$ Triton $\mathrm{X}-100), 2 \mu \mathrm{L}$ de $\mathrm{MgCl}_{2}$ (1,5 mM), 1, 5 $\mu \mathrm{L}$ de dNTP $(10 \mathrm{mM}), 1,5 \mathrm{U}$ de Taq polimerasa (Promega) y $\mathrm{H}_{2} \mathrm{O}$ destilada estéril para un volumen final de $50 \mu \mathrm{L}$. El ADN fue desnaturalizado a $95{ }^{\circ} \mathrm{C}$ por 5 minutos, seguido de 35 ciclos a $94{ }^{\circ} \mathrm{C}$ durante 1 minuto, $60{ }^{\circ} \mathrm{C} 1$ minuto y $72{ }^{\circ} \mathrm{C} 1$ minuto. Un paso de extensión final de 7 minutos a $72{ }^{\circ} \mathrm{C}$ fue añadido al final de la corrida. El producto final de la RPC fue chequeado con un marcador de peso molecular de ADN XIV (Boehringer Mannheim) que abarca un rango desde $100 \mathrm{pb}$ hasta $1.500 \mathrm{pb}$. Finalmente, se utilizó $15 \mu \mathrm{L}$ por separado de dicho producto para la digestión con las enzimas BstEII y HaeIII. Los resultados de la digestión fueron analizados mediante una electroforesis submarina en gel de agarosa al 3\% (Gibco, EUA) y se determinó la talla de cada uno de los fragmentos de restricción obtenidos por medio del marcador de peso molecular de $50 \mathrm{pb}$ (Gibco, EUA). Posteriormente, se procedió a la identificación de especies de las diferentes cepas analizadas, comparando los patrones de RPC-PLFR con las tablas reportadas por Telenti y cols ${ }^{12}$, Devallois y cols ${ }^{15}$ y la base de datos PRASITE con acceso libre (http://app.chuv.ch/prasite/index.html).

\section{Resultados}

En el presente trabajo se comparó la identificación de MNT mediante pruebas bioquímicas y la RPC-PLFR. Los resultados de las pruebas bioquímicas estuvieron disponibles entre 4 y 6 semanas a diferencia de la RPCPLFR que requirió de sólo 48 horas.

Al aplicar la RPC-PLFR en 13 cepas de referencias, se pudo apreciar concordancia de $100 \%$ en la identificación de especie. Sin embargo, la RPC-PLFR identifi- 


\begin{tabular}{|c|c|c|c|c|c|c|c|c|c|c|}
\hline \multirow[t]{2}{*}{ Especies } & \multirow[b]{2}{*}{$25^{\circ} \mathrm{C}$} & \multirow[b]{2}{*}{$37^{\circ} \mathrm{C}$} & \multirow[b]{2}{*}{$45^{\circ} \mathrm{C}$} & \multirow[b]{2}{*}{ Ureasa } & \multirow[b]{2}{*}{ Tween 80} & \multicolumn{2}{|c|}{ Aril sulfatasa } & \multicolumn{3}{|c|}{ Fuente de Carbono } \\
\hline & & & & & & 3 d & $14 d$ & inositol & manitol & Citrato \\
\hline M. avium & + & + & - & - & - & - & - & & & \\
\hline M. intracellulare & + & + & + & - & - & - & + & & & \\
\hline M. malmoense & + & + & - & + & + & - & - & & & \\
\hline M. fortuitum & + & + & - & + & + & + & & - & - & - \\
\hline M. peregrinum & + & + & - & + & + & + & & - & + & - \\
\hline
\end{tabular}

có a la cepa $M$. simiae II como $M$. simiae III, según la base de datos PRASITE (dato no mostrado).

Por otro lado, la identificación de las 46 MNT por pruebas bioquímicas evidenció la presencia de 9 especies micobacterianas, algunas de ellas, con características fenotípicas muy similares. Tales fueron los casos de las especies $M$. avium, $M$. intracellulare y $M$. malmoense, micobacterias no cromogénicas de crecimiento lento, en las que las pruebas bioquímicas de crecimiento a 25 y $45^{\circ} \mathrm{C}$, la prueba arilsulfatasa, la hidrólisis del Tween 80 y la ureasa resultaron ser decisivos para la diferenciación de estas especies (Tabla 1). Otras dos especies micobacterianas no cromogénicas pero de crecimiento rápido que resultaron ser muy similares fenotípicamente fueron las especies $M$. fortuitum y $M$. peregrinum. En este caso, la obtención de crecimiento de $M$. peregrinum en presencia de manitol fue suficiente para su identificación (Tabla 1).

La RPC-RFLP identificó 7 especies correspondientes a 9 patrones diferentes (Tabla 2). En ambos métodos, las especies $M$. intracellulare, $M$. kansasii y $M$. fortuitum resultaron ser las micobacterias más representativas de nuestro estudio (Figura 1, Tabla 2). Sin embargo, cuatro resultados discordantes fueron encontrados entre estas pruebas. Cuatro cepas, identificadas por pruebas bioquímicas como M. malmoense (2 cepas), $M$. intracellulare (1 cepa) y $M$. peregrinum (1 cepa), fueron reportadas por la RPC-PLFR como $M$. intracellulare (2 cepas), M. avium (1 cepa) y $M$. fortuitum II (1 cepa), respectivamente (Tabla 2).

\section{Discusión}

En los últimos años, numerosos métodos moleculares han sido desarrollados para la detección de diferentes especies micobacterianas. Estos métodos tienen la capacidad de reducir considerablemente el tiempo de diagnóstico e incluso, han llegado a ser

\begin{tabular}{|c|c|c|}
\hline $\begin{array}{l}\text { Número de } \\
\text { especies }\end{array}$ & $\begin{array}{l}\text { Identificación por } \\
\text { pruebas bioquímicas }\end{array}$ & $\begin{array}{l}\text { Identificación por } \\
\text { RPC-PLFR }\end{array}$ \\
\hline 14 & M. intracellulare & M. intracellulare \\
\hline 6 & M. avium & M. avium \\
\hline 1 & M. nonchromogenicum & M. nonchromogenicum \\
\hline 7 & M. kansasii & M. kansasii I \\
\hline 1 & M. kansasii & M. kansasii IV \\
\hline 1 & M. simiae & M. simiae I \\
\hline 3 & M. gordonae & M. gordonae I \\
\hline 2 & M. gordonae & M. gordonae III \\
\hline 7 & M. fortuitum & M. fortuitum II \\
\hline 2 * & M. malmoense & M. intracellulare \\
\hline 1 * & M. intracellulare & M. avium \\
\hline $1 *$ & M. peregrinum & M. fortuitum ॥ \\
\hline \multicolumn{3}{|c|}{$\begin{array}{l}\text { *Resultados discordantes entre ambos métodos } \\
\text { RPC-PLFR: Reacción en cadena de la polimerasa-polimorfismo de la longitud de los fragmen } \\
\text { tos de restricción }\end{array}$} \\
\hline
\end{tabular}

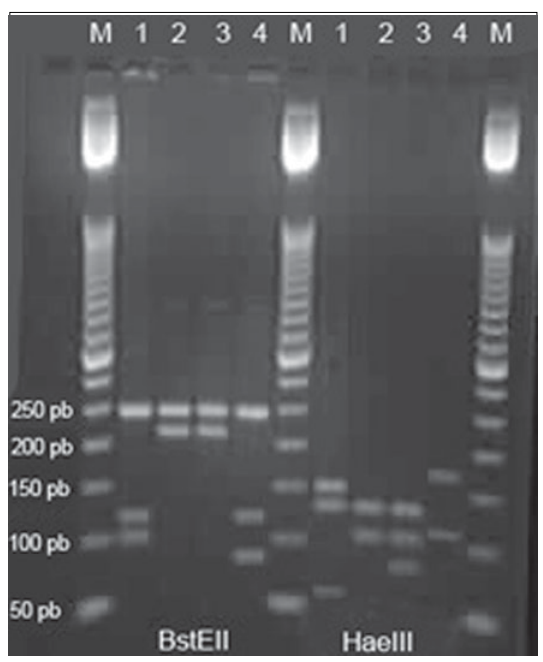

Figura 1. Resultados de los patrones de restricción de cuatro MNT al aplicar la RPC-PLFR con las enzimas BstEll y Haelll. Carril 1: M. intracellulare, Carril 2: M. avium, Carril 3: M. kansasii I, Carril 4: M. gordonae I, Carril M: Marcador de peso molecular $50 \mathrm{pb}$. 
decisivos en la identificación de nuevas especies. Una de las secuencias dianas más empleadas en estos fines es, sin duda, la comprendida dentro del gen $h s p 65$, con la cual se ha logrado la identificación de más de 50 especies de MNT, incluyendo más de $90 \%$ de las micobacterias patogénicas de crecimiento rápido, así como el diagnóstico de nuevas especies micobacterianas ${ }^{16,17}$.

En el presente trabajo se procedió a la aplicación de la RPC-PLFR utilizando 13 cepas de referencia. Sin embargo, el método falló en la diferenciación de la cepa $M$. simiae II, la cual presentó patrones similares a $M$. simiae III según la base de datos PRASITE, lo que consideramos constituye la principal desventaja de esta técnica, pues existe más de una base de datos descrita para MNT que no siempre coinciden en sus patrones reportados $2,13,16,17$. En nuestra opinión, el principal problema de la RPC-PLFR es que no se ha logrado uniformidad internacional para la interpretación de los resultados. Las diferencias pudieran deberse a condiciones de corrida aplicadas en los ensayos realizados, porcentaje de agarosa usada, e incluso, el tipo de gel empleado, puesto que se han reportado diferencias entre los patrones de corrida cuando se emplean geles de agarosa y geles de poliacrilami$\mathrm{da}^{2,16}$.

Tanto por pruebas bioquímicas como por RPC-PLFR M. intracellulare, M. kansassii y $M$. fortuitum fueron las especies más frecuentemente identificadas en esta serie que comprendió a 46 MNT; la aislada con máxima frecuencia fue $M$. intracellulare, lo cual se corresponde a un estudio similar realizado en el país durante el período 1985-1989 ${ }^{18}$. Junto con M. avium conforman el complejo Mycobacterium avium, reportado como el más frecuente en países como Brasil, E.U.A. y Canadá $^{3,19}$.

La especie $M$. kansasii ha sido también reportada como una micobacteria de importancia clínica, en especial en pacientes que sobrepasan los 50 años de edad, causando una infección pulmonar muy similar a la TBC. La incidencia de la TBC causada por esta especie depende de la región geográfica donde se realice el estudio, destacándose países como Inglaterra y España donde se han detectado un gran número de aislados de esta micobacteria ${ }^{19}$. Mycobacterium fortuitum ha sido también reconocido como una de las micobacterias de mayor incidencia en nuestro país ${ }^{20}$. En este sentido, Wong y cols realizaron un estudio en Hong Kong empleando RPC-PLFR y encontraron a $M$. fortuitum dentro de las especies más frecuentes ${ }^{20}$. Así mismo, Shulz y cols combinó la RPC anidada con enzimas de restricción para determinar la frecuencia de ADN de micobacterias en tejidos conservados en parafina por un período de tres años, destacándose el complejo Mycobacterium fortuitum, formado por las especies Mycobacterium chelonae y M. fortuitum, como la MNT mayormente encontrada ${ }^{21}$.

Cuatro cepas (de las 46 MNT analizadas) presentaron resultados discordantes al comparar los dos método de identificación empleados en nuestro estudio. Estas discrepancias también han sido encontradas por otros autores, los cuales se lo atribuyen a la presencia de más de una especie micobacteriana en el cultivo original o a errores de interpretación de las características fenotípicas y bioquímicas, dada la gran similitud que presentan estas especies en pruebas convencionales $^{4,5,22}$. Por tal motivo, trabajos publicados con anterioridad plantean que el uso de RPC-PLFR suele ser más efectivo en el diagnóstico de MNT que las pruebas bioquímicas, debido, fundamentalmente, a que los métodos convencionales se apoyan en características fenotípicas de las micobacterias, un parámetro susceptible de variar en determinadas especies, lo que pudiera conducir a un diagnóstico erróneo de las mis$\operatorname{mas}^{4,5,22}$.

En esta serie, en 5 especies micobacterianas se observaron estas discrepancias. Las pruebas bioquímicas de crecimiento a 25 y $45^{\circ} \mathrm{C}$, hidrólisis de la urea (ureasa) y del Tween 80 fueron suficientes para diferenciar las especies $M$. avium, $M$. intracellulare y $M$. malmoense. Por lo contrario, las dos cepas de $M$. malmoense detectadas por estas pruebas así como una cepa de $M$. intracellulare, fueron diagnosticadas por la RPC-PLFR como M. intracellulare (dos cepas) y $M$. avium (una cepa), respectivamente. Consideramos que estas discrepancias se deben a la estrecha similitud que presentan estas especies en las pruebas bioquímicas; no siempre el fenotipo es del todo reproducible. Por tal motivo, las tablas de identificación reportadas para MNT reflejan porcentajes o probabilidades de éxito o fracaso para una prueba determina$\mathrm{da}^{23}$. Una cepa identificada por pruebas bioquímicas como $M$. peregrinum, presentó perfiles similares a $M$. fortuitum según la RPC-PLFR. El uso de manitol como fuente de carbono resultó ser decisiva para diagnosticar la especie $M$. peregrinum, en las restantes pruebas fue muy similar a $M$. fortuitum. En este caso, además de lo planteado con anterioridad, es probable que alguna contaminación de nuestro laboratorio haya producido este diagnóstico errado para esta prueba en particular.

Las MNT presentan una relativa resistencia a un amplio espectro de fármacos antituberculosos, debido, fundamentalmente, a la poca permeabilidad y alta hidrofobia que presenta su superficie celular ${ }^{24}$. Por ello, la aplicación de un método de diagnóstico efectivo resulta crucial para ejecutar una correcta terapia específica en los pacientes. Nuestro laboratorio, como 
centro nacional de referencia del país, utiliza más de un método de diagnóstico en muestras de importancia clínica, con el objetivo de asegurar una correcta identificación $\mathrm{y}$, por consiguiente, un eficaz tratamiento. Afortunadamente, para los pacientes de nuestro estudio las discrepancias encontradas por ambos métodos no hubieran ocasionado fallas en el tratamiento de estos (de haberse realizado un solo método) ya que las infecciones ocasionadas por $M$. intracellulare, $M$. avium y $M$. malmoense son tratadas de forma similar, usualmente claritromicina o azitromicina, rifampicina y etambutol $^{24,25}$. Para infecciones ocasionadas por $M$. fortuitum y $M$. peregrinum ocurre algo similar, se ha encontrado efectividad de amikacina, ciprofloxacina, sulfonamidas y cefoxitina ${ }^{24,25}$.

En resumen, consideramos que la RPC-PLFR es un método rápido y efectivo para el diagnóstico de MNT. Su aplicación en los laboratorios de referencia pudiera ser de gran utilidad para una correcta identificación y, por ende, para el uso de una terapia adecuada en los pacientes afectados por estos microorganismos.

\section{Resumen}

La amplificación por reacción de la polimersa en cadena (RPC) de un fragmento del gen $h s p 65$, seguido del análisis del polimorfismo de la longitud de los fragmentos de restricción (PLFR) por las enzimas BstEII y HaeIII, ha demostrado ser muy útil en la identificación de micobacterias no tuberculosas (MNT). En el presente trabajo se les realizó una batería de pruebas bioquímicas así como la RPC-PLFR a un total de 13 cepas de referencia y 46 cepas recibidas en el laboratorio. Los resultados de las pruebas bioquímicas estuvieron disponibles entre 4 a 6 semanas, a diferencia de la RPC-PLFR que requirieron de sólo 48 horas. En ambos métodos, las especies detectadas con mayor frecuencia fueron Mycobacetrium intracellulare, $M$. kansasii y M. fortuitum. La RPC-PLFR es un método rápido, sencillo y eficaz. Su aplicación en los Laboratorios de Referencia pudiera ser de gran utilidad para el diagnóstico de MNT.

\section{Referencias}

1.- Hale Y M, Pfyffer G E, Salfinger M. Laboratory diagnosis of mycobacterial infections: new tools and lessons learned. Clin Infect Dis 2001; 33: 834-46.

2.- Leão S C, Bernardelli A, Cataldi A, Zumarraga M, Robledo J, Realpe T, et al. Multicenter evaluation of mycobacteria identification by PCR restriction enzyme analysis in laboratories from Latin America and the Caribbean. J Microbiol Methods 2005; 61: 193-9.

3.- Silva CF, Ueki SY, Geiger D, Leão S. hsp65 PCR-restriction enzyme analysis (PRA) for identification of mycobacteria in the clinical laboratory. Rev Inst Med Trop S Paulo 2001; 43: 120-5.

4.- Springer B, Stockman L, Teschner K, Roberts G D, Böttger E C. Two laboratory collaborative study on identification of mycobacteria: Molecular versus phenotypic methods. J Clin Mirobiol 1996; 34: 296-303.

5.- Mondragón M, Vázquez C A, Barrón C, Acosta P, Jost K C, Balandrano S, et al. Comparison among three methods for mycobacteria identification. Salud Pública México 2000; 42: 484-9.

6.- Palomino J C, Martín A, Portales F. New methods for the diagnosis and drug resistance detection in mycobacteria. Recent Res Devel Microbiol 2002; 2: 297-318.

7.- Butler W, Jost K, Kilburn J. Identification of mycobacteria by high-performance liquid chromatography. J Clin Microbiol 1991; 29: 2468-72.

8.- Lambert M A, Moss C W, U. A. Silcox U A, Good R. Analysis of mycolic acid cleavage products and cellular fatty acids of Mycobacterium species by capillary gas chromatography. J Clin Microbiol 1986. 23: 731-6.

9.- Daniel T M, Debanne S M. The serodiagnosis of tuberculosis and other mycobacterial diseases by enzyme-linked immunosorbent assay. Am Rev Respir Dis 1987; 135: 1137-51.

10.- Hance A J, Grandchamp B, Lévy-Frébault V, Lecossier D, Rauzier J, Bocart D, et al. Detection and identification of mycobacteria by amplification of mycobacterial DNA. Mol Microbiol 1989; 3: $843-9$.

11.- Plikaytis B B, Plikaytis B D, Yakrus A, Butler W R, Woodley C L, Silcox V A, et al. Differentiation of slowly growing Mycobacterium species, including Mycobacterium tuberculosis, by gene amplification and restriction fragment length polymorphism analysis. J Clin Microbiol 1992; 30: 1815-22.

12.- Telenti A, Marchesi F, Balz M, Bally F, Bottger E, Bodmer T. Rapid identification of mycobacteria to the species level by polymerase chain reaction and restriction enzyme analysis. J Clin Microbiol 1993; 31: 175-8.

13.- Vestal A L. Procedures for the isolation and identification of mycobacteria. Atlanta
(GA): Department of Health Service, CDC, 1975; 42-95.

14.- Kent P T, Kubica G P. Public health mycobacteriology: A guide for the level III laboratory. Atlanta (GA): Centers for Disease Control, US Department of Health and Public Services, 1985.

15.- Devallois A, Seg Goh K, Rastogi N. Rapid identification of mycobacteria to species level by PCR-restriction fragment length polymorphism analysis of the hsp65 gene and proposition of an algorithm to differentiate 34 mycobacterial species. J Clin Microbiol 1997; 35: 2969-73.

16.- Brunello F, Ligozzi M, Cristelli E, Bonora S, Torotoli E, Fontana R. Identification of 54 mycobacterial species by PCR-restriction fragment length polymorphism analysis of the hsp65 gene. J Clin Microbiol 2001; 39: 2799-806.

17.- Steingrube V A, Gibson J L, Brown B A, Zhang Y, Wilson R W, Rajagopalan M, et al. PCR amplification and restriction endonuclease analysis of a 65-kilodalton heat shock protein gene sequence for taxonomic separation of rapidly growing mycobacteria. J Clin Microbiol 1995; 33: 149-53.

18.- Ferrá C, Montoro E, Gutiérrez A M, Valdivia J A, Jiménez C. Estudio de micobacterias no tuberculosas aisladas en Cuba durante el período 1985-1989. Reporte preliminar. Rev Cub Med Trop 1992; 44: 205-7. 
19.- Martínez-Moragón E, Menéndez R, Palasí P, Santos M, López-Aldeguer J. Enfermedades por micobacterias ambientales en pacientes con y sin infección por el VIH: características epidemiológicas, clínicas y curso evolutivo. Arch Bronconeumol 2001; 37: 281-6.

20.- Wong D, Yip P, Tse D, Tung V, Cheung D, Kam K. Routine use of a simple low-cost genotypic assay for the identification of mycobacteria in a high throughput laboratory. Diag Microbiol Infec Dis 2003; 47: 421-6.
21.- Schulz S, Cabras A D, Kremer M, Weirich G, Miethke T, Bosmuller H C, et al. Species identification of mycobacteria in paraffinembedded tissues: frequent detection of nontuberculous mycobacteria. Mod Pathol 2005; 18: 274-82.

22.- McNabb A, Eisler D, Adie K, Amos M, Rodríguez M, Stephens G, et al. Assessment of partial sequencing of the 65 kilodalton heat shock protein gene ( $h s p 65$ ) for routine identification of Mycobacterium species isolated from clinical sources. J Clin Microbiol 2004; 42: 3000-11.
23.- Leão S, Martín A, Mejía G I, Palomino J C, Robledo J C, Telles M A, et al. Practical handbook for the phenotypic and genotypic identification of mycobacteria. Vanden Broele, Brugge, Belgium, 2004.

24.- Falkinham J O. $3^{\text {rd }}$. Epidemiology of infection by nontuberculous mycobacteria. Clin Microbiol Rev 1996; 9: 177-215.

25.- American Thoracic Society. Diagnosis and treatment of disease caused by nontuberculous mycobacteria. Am J Resp Crit Care Med 1997; 156: S1-S25. 